\title{
Ontogeny of classical and operant learning behaviors in zebrafish
}

\author{
André Valente, ${ }^{1,3,4}$ Kuo-Hua Huang, ${ }^{1,2,4}$ Ruben Portugues, ${ }^{1}$ and Florian Engert ${ }^{1,5}$ \\ ${ }^{1}$ Department of Molecular and Cellular Biology, Harvard University, Cambridge, Massachusetts 02138, USA; ${ }^{2}$ Program in \\ Neuroscience, Harvard Medical School, Boston, Massachusetts 02115, USA; ${ }^{3}$ Gulbenkian PhD Programme in Biomedicine, \\ Oeiros, 2780-156, Portugal
}

\begin{abstract}
The performance of developing zebrafish in both classical and operant conditioning assays was tested with a particular focus on the emergence of these learning behaviors during development. Strategically positioned visual cues paired with electroshocks were used in two fully automated assays to investigate both learning paradigms. These allow the evaluation of the behavioral performance of zebrafish continuously throughout development, from larva to adult. We found that learning improves throughout development, starts reliably around week 3 , and reaches adult performance levels at week 6 . Adult fish quickly learned to perform perfectly, and the expression of the learned behavior is manifestly controlled by vision. The memory is behaviorally expressed in adults for at least $6 \mathrm{~h}$ and retrievable for at least $12 \mathrm{~h}$.
\end{abstract}

[Supplemental material is available for this article.]

The ability to associate two environmental cues, as in classical conditioning, or to correlate one's behavior with its consequences, as in operant conditioning, are processes often essential for an animal's survival (Skinner 1984). While there are studies that have followed the ontogeny of learning behaviors in animal model systems of classical conditioning paradigms (Campbell and Ampuero 1985; Moye and Rudy 1985; Paczkowski et al. 1999; Raineki et al. 2009), the ontogeny of operant learning, in particular, remains much less explored.

The ability for classical conditioning is pervasive across the animal kingdom and often interpreted as the most basic and robust form of associative learning; however, it has been shown that adding an operant component to a learning task may increase performance, or show it where it was not present before (Heisenberg et al. 2001; Moore 2004).

Zebrafish is an exceptional model of vertebrate neural development and amenable both to forward genetics and genetic manipulation (Neuhauss 2003). The transparent larvae are uniquely suited to functional imaging and have recently served to uncover fundamental mechanisms in fish locomotor control (Orger et al. 2008; Portugues and Engert 2009). Behavior is the ultimate functional readout of neural activity; indeed, behavior is the ultimate evolutionary reason for the existence of brains and the changes in behavior during development should reflect the changes the nervous system undergoes as the larval organism develops into the adult. While there are well-established learning paradigms for Drosophila and rodent experimental models, zebrafish still lacks a systematic learning characterization. We describe here an approach to implement and test such a learning assay that utilizes the fact that zebrafish can see and track visual stimuli and clearly perform visually evoked locomotor behaviors as early as 5 d post-fertilization (dpf) (Easter and Nicola 1996; Budick and O'Malley 2000). To that end we use visually guided stimuli com-

\footnotetext{
${ }^{4}$ These authors contributed equally to this work.

${ }^{5}$ Corresponding author.

E-mail florian@mcb.harvard.edu.

Article is online at http://www.learnmem.org/cgi/doi/10.1101//m.025668.112.
}

bined with a noxious electroshock to control learning behaviors. We characterize these assays and specifically obtain a full ontogeny of operant learning from 7-d-old larvae to adult. This ontogeny will thus not only provide a full developmental picture of operant learning in a model vertebrate in itself but also provide a full functional correlate to the well-described development of the nervous system in zebrafish. Moreover, it will determine how early zebrafish start to be able to not just integrate and respond to visual stimuli, but significantly change their behavior as a response to visual learning.

\section{Results}

\section{Classical and operant conditioning in adult zebrafish}

To evaluate associative learning in zebrafish we set out to test two forms of learning robustly present in vertebrates, classical and operant conditioning. We developed tests that can easily be adapted and closely compared at different developmental stages from 7-dpf larvae to complete adult maturation at $8 \mathrm{wk}$. The general layout of our learning arena is described in Figure 1, A and B. Fish swim freely in a tank cued with distinct patterns that demarcate the two halves of the arena. They are monitored continuously with a video tracking system, which registers their position online at all times.

During classical conditioning (Fig. 1C) fish learn to associate an unconditioned stimulus (US), a small whole-tank electroshock, with a visual pattern that is presented beneath the tank, the conditioned stimulus (CS). The US is preceded and overlapped with the CS (as illustrated) and delivered in nine consecutive pairings at $0.1 \mathrm{~Hz}$ (Fig. 1C, pink region). This assay could also be interpreted as a form of differential classical conditioning in which one visual stimulus (the CS+ represented in Fig. $1 \mathrm{~A}-\mathrm{C}$ as a prominent checkered board) is paired with the US and another one is not (the CS - , in this case the gray panel as in Fig. 1C) (Jami et al. 2007). The animals can be trained easily to either of the stimuli. Remarkably, $2 \mathrm{~h}$ after a successful training session against the checkerboard, the same animal could be quickly conditioned 
A

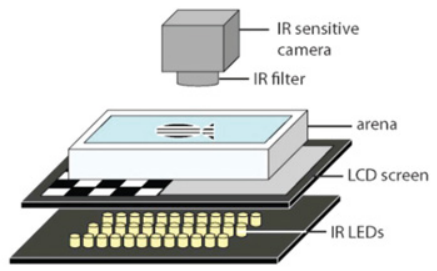

B

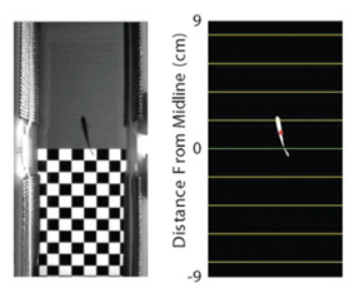

C
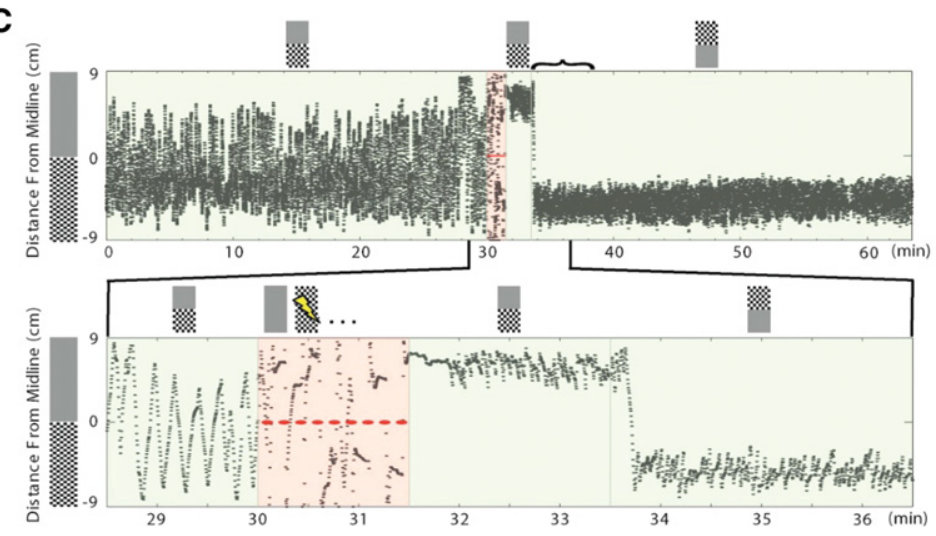

D

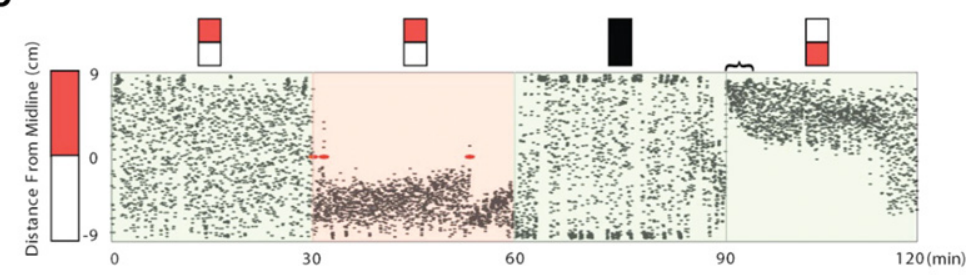

E

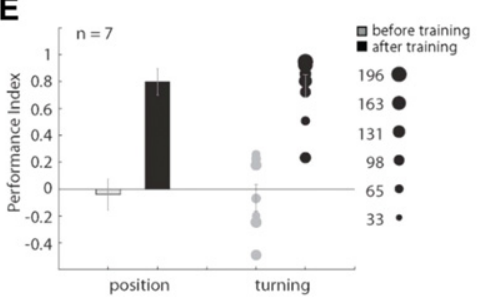

$\mathbf{F}$

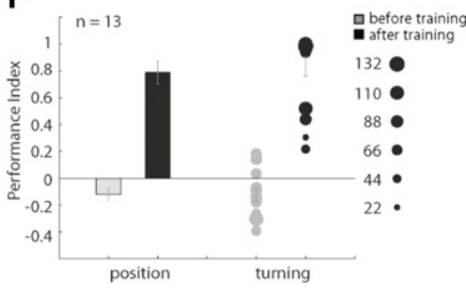

Figure 1. Classical and operant conditioning in adult zebrafish. (A) Experimental setup. The visual conditioned stimulus is presented on a LCD screen beneath an infrared-lit fish tank. The unconditioned stimulus consists of an electric shock across the arena. $(B)$ Left panel: view of the arena from above without an infrared filter; right panel: online analysis of fish location. (C) A typical example trace from a classical conditioning experiment showing the basic training protocol. (Top) Experiments start with a baseline period $(0-30 \mathrm{~min})$, followed by a training period $(30-31.5 \mathrm{~min})$, and end with a test period (31.5-64 $\mathrm{min})$. The visual cues presented at each of these stages are symbolized on top of the trace. Each black circle represents the animal's location recorded at one frame/sec. The curly bracket indicates the time window used for analyzing conditioned behavior. (Bottom) Magnified trace around the training period. Two visual cues are displayed alternatively, but only one is associated with electric shocks (red ellipses). The trained fish changes position when the visual cues are switched (31.5-36.5 min). (D) A typical example trace from an operant conditioning experiment. The assay starts with a baseline period $(0-30 \mathrm{~min})$, is followed by a training period $(30-60 \mathrm{~min})$, then a dark period (60-90 $\mathrm{min})$, and ends with a test period (90-120 min). The electric shocks applied during training are indicated by red ellipses. The conditioned response disappears immediately after visible light is removed in the dark period, but reappeared after the visual cues are presented in the test period (see Fig. 5, below). (E) Adult zebrafish show a significant learning response after classical conditioning as analyzed both by the animal's position or its turning behavior. The results of the two analyses are similar. In the turning analysis, each circle represents the performance index (PI) of a fish, and the area of the circle represents the turning numbers within the analysis window. This allows visualizing and taking into account animals that show little movement and thereby perform fewer turns. In the plot, animals represented by a smaller dot performed fewer turns in the tank. The size of the dots gives us a strong degree of confidence in the data. Animals were trained against either gray or checkerboard with comparable learning indices and data are pooled in the figure. $(F)$ Adult zebrafish show a significant learning response after operant conditioning; analysis as in $E$. against the gray stimulus as illustrated in Supplemental Movies 1 and 2.

In operant conditioning (Fig. 1D), during the $30 \mathrm{~min}$ of training, a small shock is paired with the presence of the freely moving animal in one of two visually defined halves of the tank. The distinct difference with respect to the classical conditioning assay is that here it is an aspect of the animal's own locomotor behavior, its position along the visual cues, that determines the presence of the CS and US. Training was followed by a 30-min dark period in which fish are deprived of all visual cues.

Both forms of learning are tested by monitoring the behavior of fish in the presence of the paired and unpaired (conditioned and neutral) visual cues (Fig. 1C,D, second half). The spatial preference of the fish is analyzed either based on their recorded position or on the number of times fish turn away from the conditioned cues. In the data shown in Figure 1, D and F, fish were conditioned against red. They can be equally conditioned against the white stimulus (Supplemental Fig. 1). Both positional and turning analyses clearly show that adult fish can learn these tasks extremely well (Fig. 1E,F), avoiding swimming in the areas paired with the noxious stimuli with performance indices (PIs) close to +1 .

If visuo-spatial stimuli are reversed during testing, the animal's preferred position reverses accordingly. In Figure 1, C and $\mathrm{D}$, the vertical lines at 33.5 and $90 \mathrm{~min}$, respectively, indicate the time point at which the visual cues are reversed (symbolic icons at top).

\section{Ontogeny of learning in the classical conditioning assay}

To assess when zebrafish first start expressing learning behaviors, we continuously tested the same group of fish in our classical conditioning assay from larval to adult stages (see Fig. 2A-C). Tank and visual stimulus size was scaled to fish stage in order to provide similar learning conditions to all animals. Figure 2D depicts the performance index of an individual group of fish that underwent classical conditioning for five consecutive days every week, over a period of $>6 \mathrm{wk}$.

The freely swimming group of fish was briefly exposed to a visual pattern paired to precede and overlap with a whole-tank electroshock for nine consecutive pairings at $0.1 \mathrm{~Hz}$ (Fig. 1C, pink region). Figure $2 \mathrm{D}$ shows the performance index of the animals after training as they mature to adults. It is apparent that fish start learning significantly at 

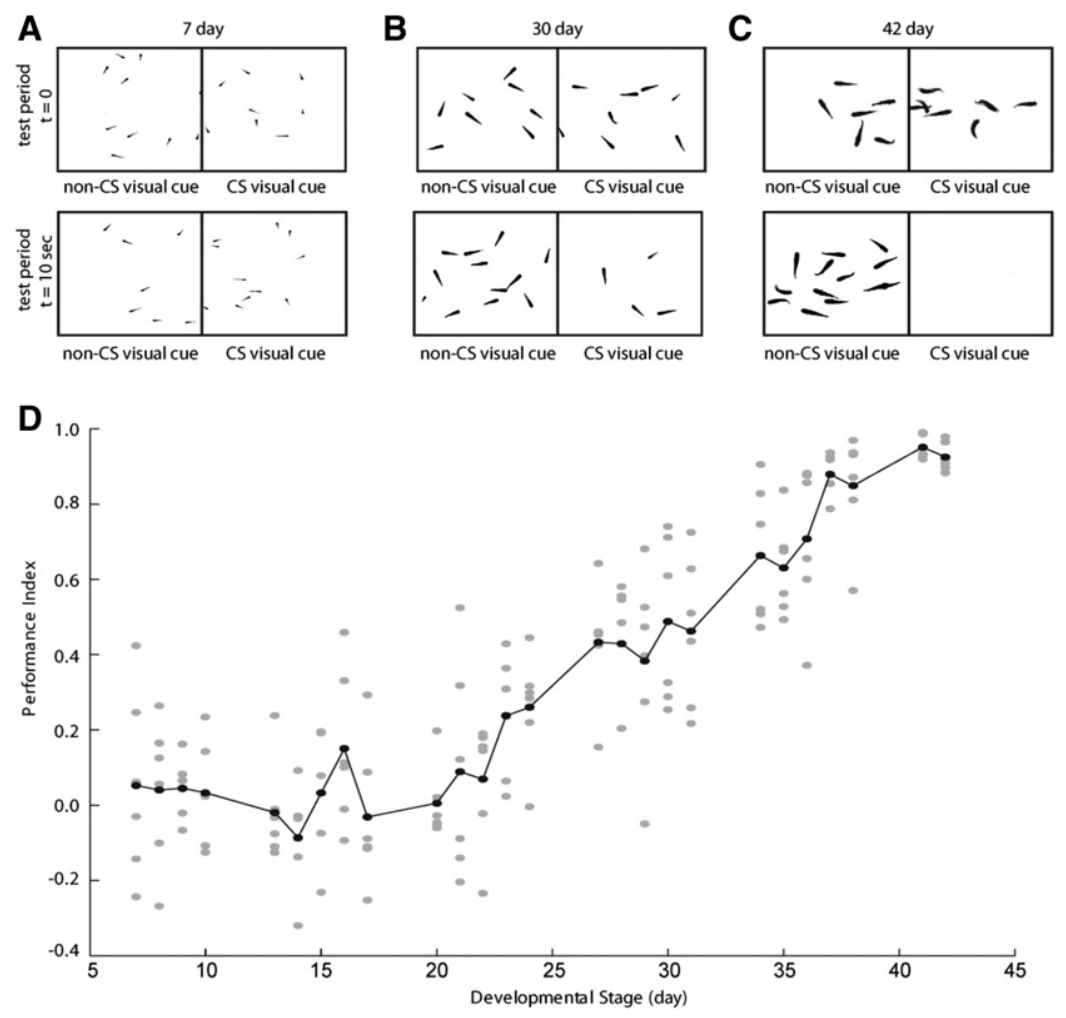

Figure 2. Ontogeny of learning in the classical conditioning assay. $(A-C)$ Typical example responses of 7-, 30-, and 42-d-old fish to the presence of visual CS. As fish mature, they progressively show a clear response, distributing themselves to avoid the CS during testing. $(D)$ In our classical conditioning assay, learning is significant at week 4 and reaches adult performance level at week 6 . The animals are trained and tested five times per day, and their performance is quantified based on their position in the arena.

week 4 and reach close to the maximum PI by week 6 . As seen in Figure 1, this is maintained in older adults. While the data gathered with this assay are striking and informative, there are several caveats with this experiment. Firstly, fish are trained in groups, which may lead to interference effects between animals. Secondly, the repeated training sessions, which have been chosen for maximum impact, also contain inherently repeated extinction trials, which make the results harder to interpret. These compromising factors might serve as an explanation of why fish apparently start learning only at an age $>25 \mathrm{~d}$. Finally, as mentioned in the introduction section, adding an operant component is known to increase the performance, and the experiment shown here relies mostly on a classical assay. Therefore, we subsequently tested individual animals in an operant assay to investigate the ontogeny of learning.

\section{Ontogeny of learning in the operant conditioning assay}

We analyzed operant conditioning over the course of development by assaying different individual fish, each taken naively at different stages, from larva to adult. In this assay, we observed that the capacity to learn begins at week 3 and reaches the maximum PI of +1 for almost all individuals tested by week 6 , which is maintained in adult performance (Fig. 3D). Even though we established a common training period of $30 \mathrm{~min}$, it is evident that the number of shocks fish expose themselves to decreases as they develop, that is, they take progressively fewer shocks to learn to avoid the paired stimulus (Fig. 3E). Even between weeks 7 and 8, when their performance level appears indistinguishable, there is a significant decrease in the number of shocks fish expose themselves to during training. This number decreases to less than half, from an average of 19 to seven shocks ( $t$-test, $P=0.0272$ ) per training session.

There is substantial individual variability in results before week 3 , indicating that some larvae could be able to perform these tasks. Although there is a progressive decrease in the number of shocks individuals expose themselves to, the decrease only becomes significant between weeks 2 and 3, when the performance index also becomes significantly higher ( $t$-test, $P=0.0492$ ).

\section{Persistence of memory in behavior of adult zebrafish}

To assess the persistence of the learned behavior in adults we tested for the presence of conditioned behavior in response to the visual cues after an increasing length of time in the absence of visual stimuli after operant training.

Visual stimuli with reversed orientation were introduced after a period of no light in the tank to ensure that the conditioned behavior is based on the presented visual stimuli rather than other nonvisual or distal visual cues (Fig. 4A, example trace at $90 \mathrm{~min}$ ). We found that the conditioned response disappears immediately after the removal of light, indicating that the behavior was purely visually mediated (Figs. 1D, 4A; 60-90 min, dark icon).

To test for the permanence of the memory in behavior, the length of the period with no light was gradually increased before reintroduction of the visual cues. Figure $4 \mathrm{~B}$ plots the performance index observed for increasing periods in the absence of light. Figure 4A serves as an example of typical raw trace, where the positional distribution of the animal after the reintroduction of the stimulus is clearly biased toward the unpaired visual stimulus in spite of the reversal of orientation of the stimuli presented (Fig. $4 \mathrm{~A}, t>90 \mathrm{~min})$.

In this assay, the memory is shown to control the fish's behavior, showing a PI significantly different from zero, for at least $6 \mathrm{~h}$ (Fig. 4B). By $12 \mathrm{~h}$, fish have on average apparently lost the conditioned behavior (although high variability is seen here).

The apparent decrease in average PI to zero observed after $12 \mathrm{~h}$ in the absence of light (Fig. 4B) could mean that most animals have by this time positively lost the conditioned memory, that they are less responsive to stimulus reintroduction as this comes during the nighttime of their light-dark cycle, or, alternatively, that this memory is still represented in the nervous system, though no longer dominating the animals' behavior in response to the conditioned visual stimulus. In order to test for the ability to effectively recall this memory, we provided a shock 30 min after testing (red dot at $120 \mathrm{~min}$ in Fig. 4A). It is clear that in most animals a brief electric shock can reinstate the behavior even after a 12-h lag. We performed an additional experiment with $24 \mathrm{~h}$ in the absence of light to test whether reintroduction of the visual cues during the dark phase of their cycle could be showing an effect of sleep in the 12-h time point and observed a similar result; the animals do not show significant conditioned behavior. 

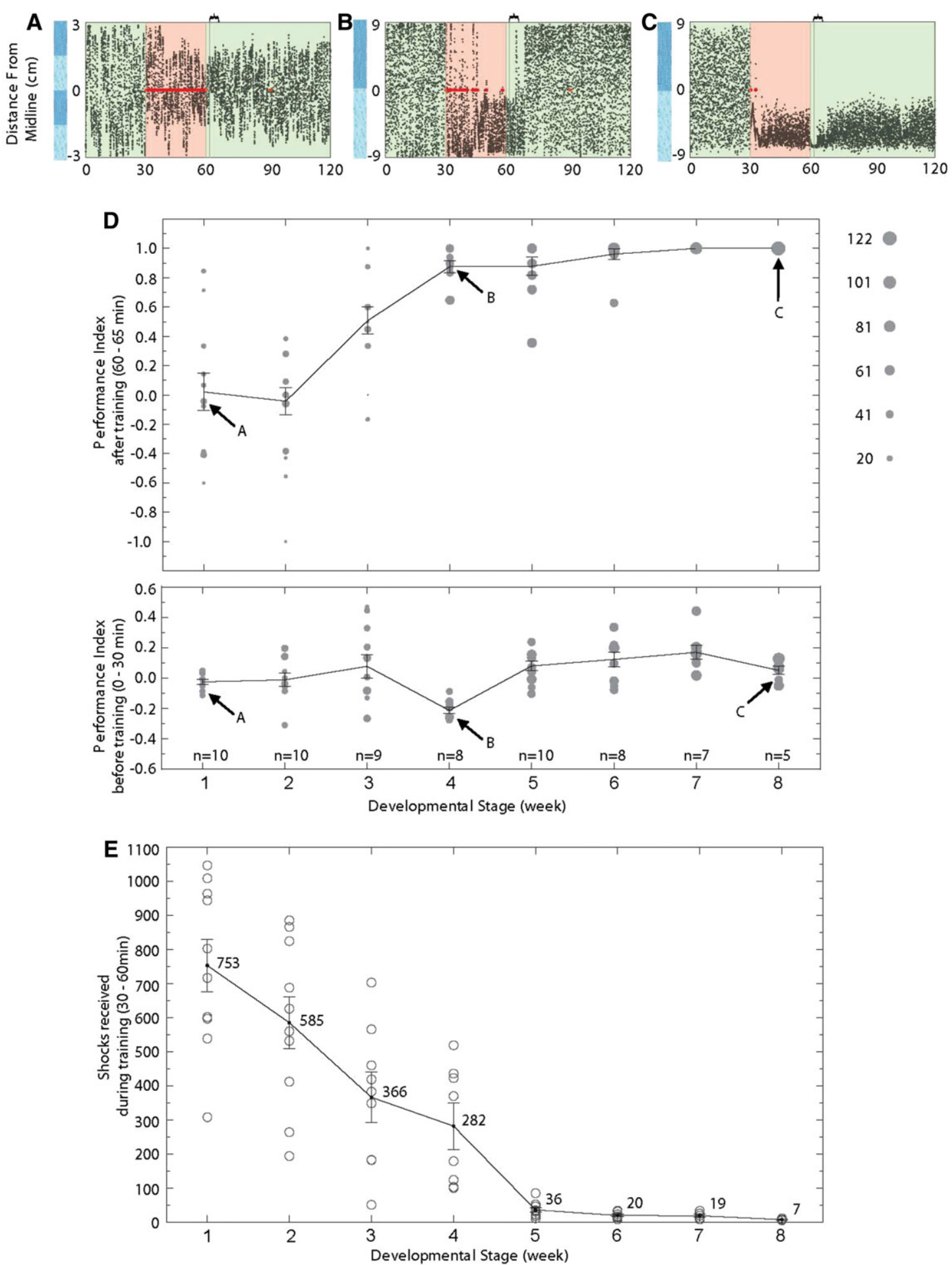

Figure 3. Ontogeny of learning in the operant conditioning assay. $(A-C)$ Example traces of the operant conditioning assay at different developmental stages. The baseline period ( $0-30 \mathrm{~min})$ is followed by a training period $(30-60 \mathrm{~min})$ and ended with a test period $(60-120 \mathrm{~min})$. The visual cues are indicated on the left and maintained throughout the experiments. Striped visual cues are used for fish younger than 4 wk to increase their chance of encountering the borderlines. The red dots indicate the shock events in the training period. The curly bracket indicates the time window for analysis. $(D$, top $)$ Conditioned behavior at different developmental stages. Each circle represents the performance index (PI) of a fish. The area of the circle is proportional to the turning events during the analysis window. Arrows indicate the PI of the fish demonstrated in $A-C$. A consistent learning behavior in zebrafish emerges in week 3 and reaches adult performance during the juvenile stage, and specifically at week 6 . The animals are trained and tested individually, and naive animals are used at each developmental stage. ( $D$, bottom) Zebrafish show no innate preference for the visual stimuli at all developmental stages. (E) Number of shocks received during the training period (30-60 min) at each developmental stage assayed. The number of shocks decreases throughout development, even after week 4 when the PI is already close to 1 . The number of shocks fish expose themselves to shows a significant decrease between weeks 2 and 3,4 and 5 , and 7 and 8 ( $t$-test, $P=0.0492, P=0.0004$, and $P=0.0272$, respectively). 
A

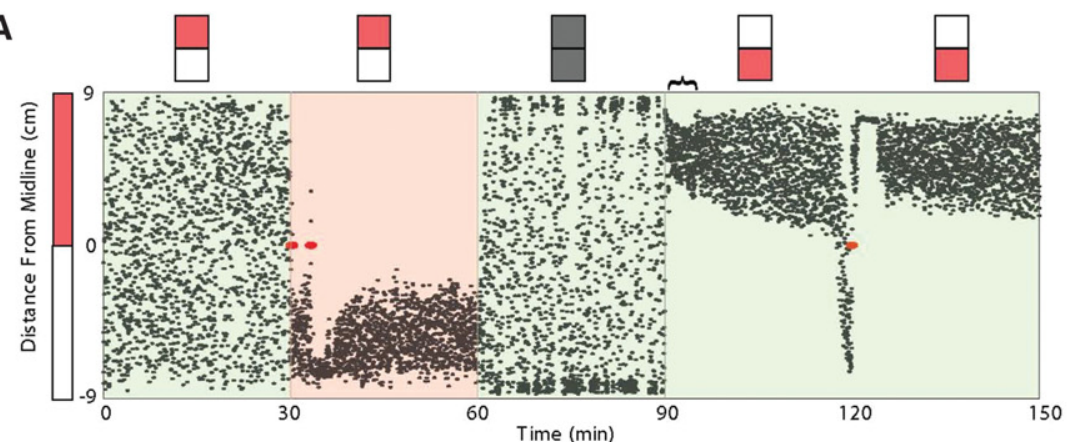

B

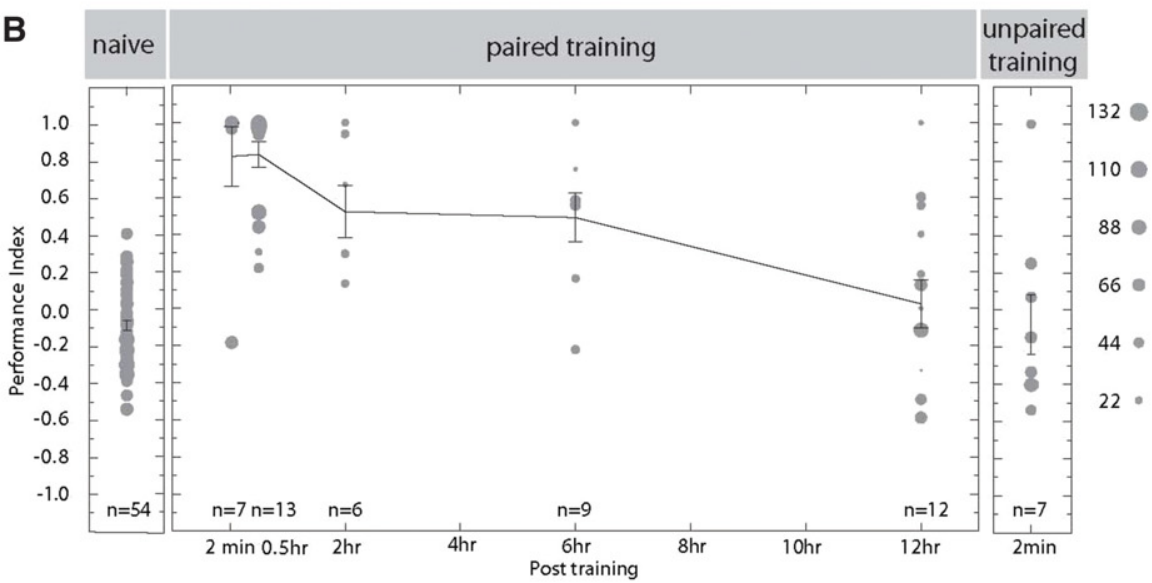

C

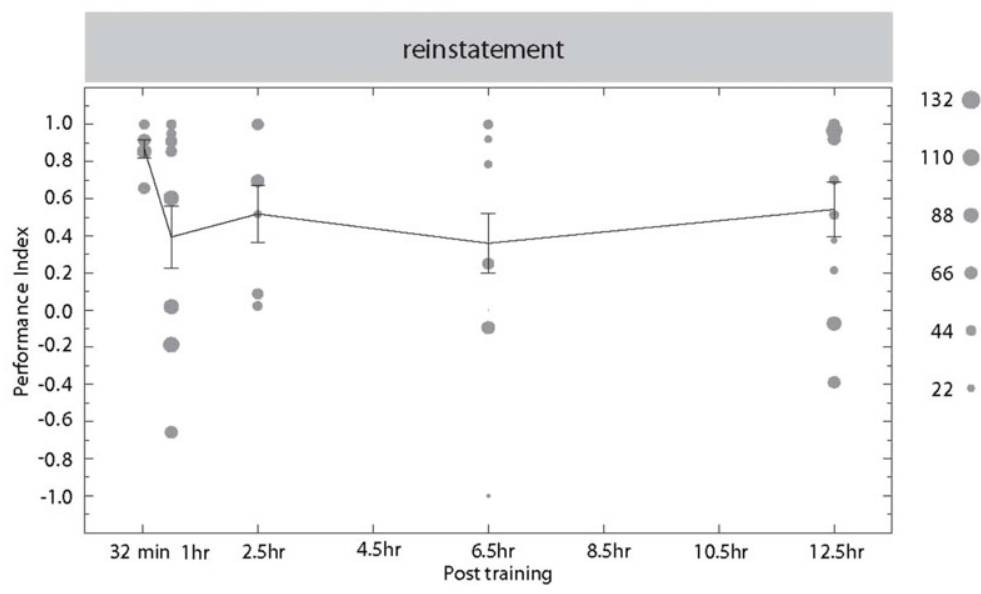

Figure 4. Persistence of memory in adult zebrafish. Persistence of conditioned response after an increasing length of dark period. Fish are trained and tested individually, and a new fish is used in each condition. Turning performance analysis calculated unless otherwise indicated. (A) An example trace of an operant conditioning experiment involving a 30-min dark period (60-90 min) between the training (30-60 $\mathrm{min})$ and test period $(90-120 \mathrm{~min})$. Each dot represents the animal's location in the arena. The red ellipse in the training period represents the shock event. This animal expresses a robust conditioned response $30 \mathrm{~min}$ after learning. ( $B$, left) Naive adult zebrafish show no innate preference to the visual stimuli. (Middle) The conditioned response is largely intact $30 \mathrm{~min}$ after learning and gradually decreases until a residual response at $6 \mathrm{~h}$ after training. (Right) Seven fish serve as a yoke experiment, receiving the electroshocks applied to the paired training group (2-min dark period). These shocks were uncorrelated to the fish's own location, and the animals show no significant learning behavior. (C) A whole-tank electric shock leads to re-expression of conditioned memory in behavior. After 30 min of testing in the presence of visual cues after different lengths of time in the dark $(90-$ 120 min as example shown in $A$ ) fish are given a single electroshock in the middle of a 20 -sec period with light removed (to hinder direct association with visual stimuli). Fish that had apparently lost the expression of memory from behavior after $12 \mathrm{~h}$ in the absence of light show a recovered performance index.
However, after a reinstatement shock, four of the eight individuals tested show a learning performance index, indicating that even if there is high variability, the memory may still be present in many individuals after this time (Supplemental Fig. 2).

\section{Vision is the sensory modality governing the expression of the learned behavior}

The learned spatial avoidance is expressed solely in the presence of visual cues and disappears completely when these are removed (Fig. 5A). Locomotion is maintained and increased in the absence of visual cues and thus a decrease in PI is not confounded by fish ceasing activity (Fig. 5B). In particular, when the visual cues are reintroduced to fish kept in the dark, animals almost immediately return to their learned performance (Fig. 5C) while maintaining their average locomotor activity in the tank (Fig. 5D). The presence of other sensory modalities such as audition and lateral line sensing do not contribute to or are insufficient for the expression of the learned behavior.

\section{Discussion}

Here, we provide a close analysis of the development of both classical and operant learning behaviors in zebrafish. We characterized the complete ontogeny of a learning behavior in a vertebrate model system from the time larvae first exhibit controlled visually elicited behaviors to full adult maturation. Results from our assays show that the ability to learn is first expressed in a window of time from 3 wk post-fertilization.

Ontogenies of classical conditioning in model systems have been intensely studied (Moye and Rudy 1985; Paczkowski et al. 1999), and more recently with advances in molecular and developmental neurobiology, these ontogenies have taken on an important role in serving as functional readouts for the underlying neural circuitry as it develops and progressively assembles components required for behavior and plasticity. The possibility of real time in vivo imaging of neural development and activity in zebrafish makes this particular model system especially attractive (Niell et al. 2004).

Our results show remarkable similarity to those observed in mammals: Rats and mice also show a defined window of time in which conditioning can 
A Entering Dark Period

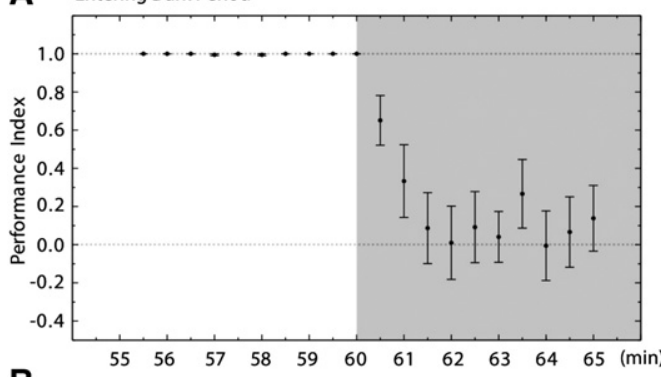

B

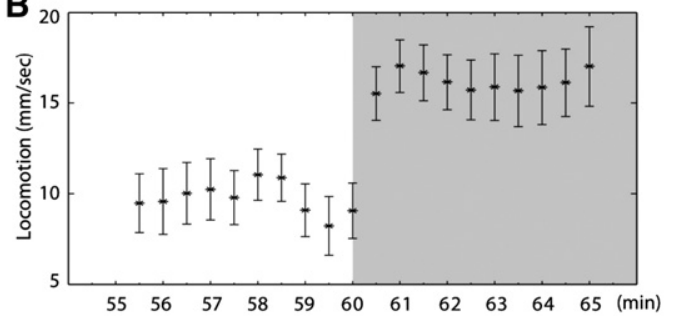

C Leaving Dark Period
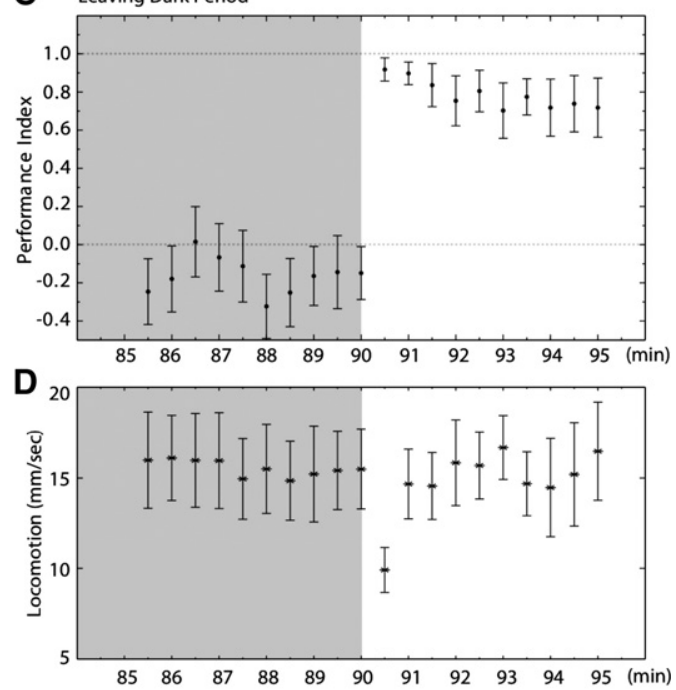

Figure 5. Vision is the sensory modality governing the expression of the learned behavior. (A) Performance index of fish 5 min before and after removal of light. Animals used in operant conditioning experiment in Figure 1F. When the dark period is instated, fish quickly redistribute their movement to the whole tank, as can be seen by the decrease in the performance index. (B) Locomotion of fish during the same period registered in $A$. At the end of the training period animals are moving constantly while expressing the learned behavior, and this transition to the dark period results in increased swimming. (C) Performance index of fish $5 \mathrm{~min}$ before and after visual stimuli are reintroduced. Reintroducing the visual cues results in the memory being reexpressed, as can be seen by the increase in the performance index. (D) The change observed upon light reintroduction in $C$ is achieved while retaining the same mobility in the tank.

first be induced. This window (generally occurring between 2 and 3 wk after birth) is common for many different paradigms such as different forms of Pavlovian fear conditioning and eyeblink conditioning (Campbell and Ampuero 1985; Moye and Rudy 1985; Paczkowski et al. 1999; Raineki et al. 2009). Moreover, studies of Pavlovian fear conditioning in infant hooded rats have found a dissociation in time between the ability to first detect visual events and to use these for associative learning (Moye and Rudy 1985).

To date there are no studies focusing on the initial development and maturation of learning behaviors in zebrafish. Studies have shown that adult zebrafish are good learners, both using food as an appetitive reward (Williams et al. 2002; Colwill et al. 2005) and noxious electroshock stimuli (Pradel et al. 1999; Rawashdeh et al. 2007; Xu et al. 2007; Blank et al. 2009), some of these uncovering potential anatomical pharmacological and neurochemical components of the system where learning occurs (Pradel et al. 2000; Xu et al. 2007; Blank et al. 2009; Jesusathan 2011; Okamoto et al. 2011). We have developed computer-based automated behavioral assays which we can easily control and comprehensively analyze. In our assays, adult zebrafish are clearly shown to be excellent learners, as in both assays animals create a robust memory after only brief exposure to the unconditioned stimulus-conditioned stimulus (US-CS) pairing. Training and testing can be done without removing the animal from the water during the post-training period, and the assay can easily be upgraded to monitor large numbers of animals at the same time for high-throughput analyses.

We have used two different classical and operant-conditioning freely swimming behavioral assays. Despite the differences between these assays, both show a similar time window for learning onset in development. Even though most zebrafish larvae performed poorly in both assays, the systems involved in visual detection and locomotor response are known to be fully functional at $7 \mathrm{dpf}$. Larval zebrafish at $5 \mathrm{dpf}$ already express robust visually induced behaviors, including the optokinetic response (OKR)
(Brockerhoff et al. 1995; Easter and Nicola 1996; Portugues and Engert 2009), phototaxis (Burgess and Granato 2007), the optomotor response (OMR) (Clark 1981; Orger et al. 2000), and prey capture (Budick and O'Malley 2000; Borla et al. 2002; Gahtan et al. 2005). In several of these studies the locomotor behavior of larval zebrafish was characterized in detail and included multiple swimming and turning components that can be assembled together to generate very fine motor control as observed, for example, during prey capture. Larval zebrafish can perform visual tracking and fine optomotor responses even when immobilized in agar, and in such experimental preparation also failed to show classical conditioning (Supplemental Fig. 3). Nonetheless, it is possible that the visual system of larval zebrafish is not mature enough to distinguish between the different stimuli in our assay. We deliberately avoided the use of stimuli that elicit any innate preference or aversion, as these make the interpretation of the assay inherently difficult. Together, this indicates that larval zebrafish between weeks 1 and 2 already have a functional visual system and control of locomotion, even though these sensorimotor capabilities may not yet have matured sufficiently to reliably support learning in our conditioning assays. Some of the larvae tested did show high learning performance indices (Fig. 3D), and recent data suggests that larval zebrafish are able to associate visual cues and tactile noxious stimuli (Aizenberg and Schuman 2011).

\section{Materials and Methods}

\section{Animals and apparatus}

In all experiments, where sexual dimorphism was clear, only female zebrafish were used to avoid any possible sexual bias in behavior (Dalla and Shors 2009). Zebrafish of strain AB swam freely in a custom-built acrylic tank with opaque walls and a transparent bottom. The tank's size is $6 \mathrm{~cm} \times 6 \mathrm{~cm} \times 2.5 \mathrm{~cm}$, with water 1-cm deep for animals younger than $3 \mathrm{wk}$ (Gahtan et al. 2005), and $18 \mathrm{~cm} \times 6 \mathrm{~cm} \times 12 \mathrm{~cm}$, with water 3 -cm deep for older fish. Visual stimuli are presented on a LCD screen immediately below 
the tank (Samsung SyncMaster, 15 inch, $1024 \times 768$ pixels). Swimming behavior is recorded at one frame per second using an infrared-sensitive CCD camera positioned above the tank. A 15-volt Infrared LED array was custom built within the LCD screen to illuminate the arena from below. An infrared filter is positioned in front of the camera to block visible light. This facilitates online analysis of fish position via a custom-written LabView program (National Instruments). A small fan is used to dissipate the heat generated by IR-LEDs. Electric shocks $(70 \mathrm{msec}, 9 \mathrm{~V} / 6 \mathrm{~cm})$ are delivered via four pieces of steel mesh, two on each side of the arena. Shock delivery at each side of the arena is controlled independently. Dose response curves to strength of electric stimuli were obtained (data not shown), and we chose stimulus values around $9 \mathrm{~V}$ that were placed in the range of values that elicits a response in all stages with $100 \%$ reliability and no detectable short or longterm damage to the animal. Experiments were undertaken at daytime in the animals' $14-10 \mathrm{~h}$ light-dark cycle (L: 9 am-11 pm; D: $11 \mathrm{pm}-9 \mathrm{am})$. In the experiments in Figure 4, memory retention after $12 \mathrm{~h}$ was assayed at nighttime.

\section{Training protocols}

All behavioral experiments are divided into baseline, training, and test periods. Fish are introduced to the arena $30 \mathrm{~min}$ before starting experiments with the LCD screen on, with the cues to be used for conditioning, Supplemental Figure 4 (no innate preference is shown for any of the cues used as shown in the results for the naive period).

During classical conditioning, in the baseline and test periods, the two distinct visual cues are presented below the tank, and no electric shocks are delivered. During training, the conditioned visual stimulus (CS) is presented for $1.5 \mathrm{sec}$, followed by the nonconditioned visual stimulus (non-CS) for $8.5 \mathrm{sec}$, nine times, for $1.5 \mathrm{~min}$. An electric shock (70 msec, $9 \mathrm{~V} / 6 \mathrm{~cm}$ ) serves as unconditioned stimulus (US), which overlaps and ends simultaneously with the CS visual cue.

For the experiments described in Figure 1, C and E, individual naive 1-yr-old fish were used. For the experiments described in Figure 2, 17 fish were trained and tested as a group starting at $7 \mathrm{dpf}$. This group was used repetitively throughout the experiment up to $42 \mathrm{dpf}$. In this experiment fish were trained as in the protocol above, but for six consecutive times, with test periods of 90 sec between.

During operant conditioning experiments, the design for the baseline and test periods is the same as in the classical conditioning assay. During operant training, the two visual cues are presented simultaneously below the tank, and electric shocks are delivered at frame acquisition rate $(1 \mathrm{~Hz})$ only whenever the animal enters the area demarcated by the conditioned visual cue. This is followed by a dark period of variable length and subsequent reverse cue introduction. Cues are always reintroduced for testing when the animal is swimming over the area corresponding to non-CS. For the experiments described in Figure 1, D and F, individual naive 1-yr-old fish were used. For those described in Figure 3 , individual naive fish were trained and tested at each developmental stage. Training was performed for both the different cues used in each experiment to control for any innate preference. In addition, cues of similar luminance were used for larvae and juveniles to prevent bias from the strong phototaxis response present in larvae. The cues used for the experiments are included in Supplemental Figure 4.

In the experiments that test the persistence of memory, a period during which all visible lights are turned off, is introduced between training and test periods. A whole-tank reinstatement shock is provided after $30 \mathrm{~min}$ of test; this shock is presented in the middle of a 20 -sec period of no light in the tank so as to avoid pairing with any particular visual cue.

\section{Analysis}

The swimming behavior along the tank is quantified based on either the animal's position or its turning behavior. In positional analysis, the animal receives a score of -1 or +1 in each image frame if its center of mass is in the conditioned or nonconditioned visual area of the tank, respectively. The performance index (PI) is the average of the scores in the first 5 min of the test period. An animal that only swims in the non-CS area during the time window shows a PI of +1 , a fish that swims evenly between the two zones shows a PI of zero and, conversely, a fish that would only swim in the conditioned area would have a PI of -1 .

In turning behavior analysis, an animal receives a score of +1 if it turns away from the CS visual cue, and receives -1 if it turns away from the non-CS visual cue. The animal receives a score of zero if it turns at the two ends of the tank. The PI is the sum of scores divided by the half number of all turning events during the analysis window. Thus, an animal that always turns away from the CS visual cue receives a PI of +1 . A fish that only turns at the end of the tank, a behavior that is often observed in naive animals, receives a score of zero. The PI of a fish in the figures is represented by a circle. The center represents the value of the PI and the area is proportional to the overall number of turning events. The analysis of turning behavior, therefore, weighs the performance of fish according to their total locomotion. In locomotion analysis we analyzed the movement of the fish at a $5-\mathrm{Hz}$ frame rate. We calculated speed from the difference in position every second and plotted the average for every $30 \mathrm{sec}$ in Figure 5. $T$-tests are performed where necessary, always assuming unequal variance.

\section{Acknowledgments}

Funding for this study was provided by the Department of Molecular and Cellular Biology, Harvard University; the Bial Foundation (115/04); the US National Institutes of Health (R01 EY014429-01A2); and the Fundação para a Ciência e Tecnologia (SFRH/BD/11767/2003). We thank all of the members of the Engert Lab, Adam Kampf, Michael Orger, John Dowling, Margarida Ramos, and Marta Moita for critical insights and discussions and Florin Albeanu for advice on experimental techniques.

\section{References}

Aizenberg M, Schuman E. 2011. Cerebellar-dependent learning in larval zebrafish. J Neurosci 31: 8708-8712.

Blank M, Guerim LD, Cordeiro RF, Vianna MR. 2009. A one-trial inhibitory avoidance task to zebrafish: Rapid acquisition of an NMDA-dependent long-term memory. Neurobiol Learn Mem 92: 529-534.

Borla MA, Palecek B, Budick S, O'Malley DM. 2002. Prey capture by larval zebrafish: Evidence for fine axial motor control. Brain Behav Evol 60: $207-229$.

Brockerhoff SE, Hurley JB, Janssen-Bienhold U, Neuhauss SC, Driever W, Dowling JE. 1995. A behavioral screen for isolating zebrafish mutants with visual system defects. Proc Natl Acad Sci 92: 10545-10549.

Budick SA, O'Malley DM. 2000. Locomotor repertoire of the larval zebrafish: Swimming, turning and prey capture. J Exp Biol 17: 2565-2579.

Burgess HA, Granato M. 2007. Modulation of locomotor activity in larval zebrafish during light adaptation. J Exp Biol 210: 2526-2539.

Campbell BA, Ampuero MX. 1985. Dissociation of autonomic and behavioral components of conditioned fear during development in the rat. Behav Neurosci 99: 1089-1102.

Clark DT. 1981. Visual responses in developing zebrafish. University of Oregon Press, Eugene, OR.

Colwill RM, Raymond MP, Ferreira L, Escudero H. 2005. Visual discrimination learning in zebrafish (Danio rerio). Behav Process 70: 19-31.

Dalla C, Shors TJ. 2009. Sex differences in learning processes of classical and operant conditioning. Physiol Behav 97: 229-238.

Easter SS Jr, Nicola GN. 1996. The development of vision in the zebrafish (Danio rerio). Dev Biol 180: 646-663.

Gahtan E, Tanger P, Baier H. 2005. Visual prey capture in larval zebrafish is controlled by identified reticulospinal neurons downstream of the tectum. J Neurosci 25: 9294-9303.

Heisenberg M, Wolf R, Brembs B. 2001. Flexibility in a single behavioral variable of Drosophila. Learn Mem 8: 1-10.

Jami SA, Wright WG, Glanzman DL. 2007. Differential classical conditioning of the gill-withdrawal reflex in Aplysia recruits both NMDA receptor-dependent enhancement and NMDA receptordependent depression of the reflex. J Neurosci 27: 3064-3068. 
Jesusathan S. 2011. Fear, anxiety and control in the zebrafish. Dev Neurobiol doi: 10.1002/dneu.20873.

Moore BR. 2004. The evolution of learning. Biol Rev Camb Philos Soc 79: $301-335$.

Moye TB, Rudy JW. 1985. Ontogenesis of learning: VI. Learned and unlearned responses to visual stimulation in the infant hooded rat. Dev Psychobiol 18: 395-409.

Neuhauss SC. 2003. Behavioral genetic approaches to visual system development and function in zebrafish. J Neurobiol 54: 148-160.

Niell MN, Meyer MP, Smith SJ. 2004. In vivo imaging of synapse formation on a growing dendritic arbor. Nat Neurosci 7: 254-260.

Okamoto H, Agetsuma M, Aizawa H. 2011. Genetic dissection of the zebrafish habenula, a possible switching board for selection of behavioral strategy to cope with fear and anxiety. Dev Neurobiol doi: 10.1002/dneu.20913.

Orger MB, Smear MC, Anstis SM, Baier H. 2000. Perception of Fourier and non-Fourier motion by larval zebrafish. Nat Neurosci 3: $1128-1133$.

Orger MB, Kampff AR, Severi KE, Bollmann JH, Engert F. 2008. Control of visually guided behavior by distinct populations of spinal projection neurons. Nat Neurosci 11: 327-333.

Paczkowski C, Ivkovich D, Stanton ME. 1999. Ontogeny of eyeblink conditioning using a visual conditional stimulus. Dev Psychobiol 35: $253-263$.
Portugues R, Engert F. 2009. The neural basis of visual behaviors in the larval zebrafish. Curr Opin Neurobiol 19: 644-647.

Pradel G, Schachner M, Schmidt R. 1999. Inhibition of memory consolidation by antibodies against cell adhesion molecules after active avoidance conditioning in zebrafish. J Neurobiol 39: 197-206.

Pradel G, Schmidt R, Schachner M. 2000. Involvement of L1.1 in memory consolidation after active avoidance conditioning in zebrafish. $J$ Neurobiol 43: 389-403.

Raineki C, Shionoya K, Sander K, Sullivan RM. 2009. Ontogeny of odor-LiCl vs. odor-shock learning: Similar behaviors but divergent ages of functional amygdala emergence. Learn Mem 16: 114-121.

Rawashdeh O, de Borsetti NH, Roman G, Cahill GM. 2007. Melatonin suppresses nighttime memory formation in zebrafish. Science 318: 1144-1146.

Skinner BF. 1984. The evolution of behavior. J Exp Anal Behav 41: $217-221$.

Williams FE, White D, Messer WS. 2002. A simple spatial alternation task for assessing memory function in zebrafish. Behav Process 58: $125-132$

Xu X, Scott-Scheiern T, Kempker L, Simons K. 2007. Active avoidance conditioning in zebrafish (Danio rerio). Neurobiol Learn Mem 87: 72-77.

Received January 12, 2012; accepted in revised form February 7, 2012. 


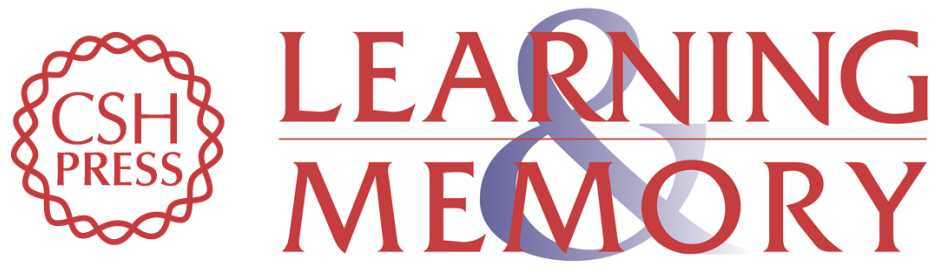

\section{Ontogeny of classical and operant learning behaviors in zebrafish}

André Valente, Kuo-Hua Huang, Ruben Portugues, et al.

Learn. Mem. 2012, 19:

Access the most recent version at doi:10.1101//m.025668.112

Supplemental http://learnmem.cshlp.org/content/suppl/2012/03/20/19.4.170.DC1

Material

References This article cites 30 articles, 8 of which can be accessed free at: http://learnmem.cshlp.org/content/19/4/170.full.html\#ref-list-1

License

Email Alerting Receive free email alerts when new articles cite this article - sign up in the box at the Service top right corner of the article or click here. 